\title{
Validity of Autotaxin as a Novel Diagnostic Marker for Liver Fibrosis in Egyptian Chronic HCV Patients
}

\author{
Wafaa M. Ezzat ${ }^{1 *}$, Halla M. Ragab², Nabila Abd El Maksoud ${ }^{2}$, Nour A. Abdulla ${ }^{3}$, Yasser A. Elhosary ${ }^{1}$ \\ ${ }^{1}$ Internal Medicine Department, National Research Center, Cairo, Egypt; ${ }^{2}$ Biochemistry Department, Genetic Engineering \\ and Biotechnology Division, National Research Center, Cairo, Egypt; ${ }^{3}$ Hepatology Department, National Hepatology \& \\ Tropical Medicine Research Institute, Cairo, Egypt
}

\begin{abstract}
Citation: Ezzat WM, Ragab HM, El Maksoud NA, Abdulla NA, Elhosary YA. Validity of Autotaxin as a Novel Diagnostic Marker for Liver Fibrosis in Egyptian Chronic HCV Patients. OA Maced J Med Sci. 2013 Dec 15; 1(1):21-26. http:/dx doi org/10.3889/oamims.2013.005

Key words: Autotoxin (ATX); HCV; liver fibrosis.

"Correspondence: Wafaa M Ezzat. Assist. Prof. of Tropical Medicine. National Research Center. EI bohooth st., Dokki, Giza, Egypt. E-mail: Wafaa 3t@yahoo.com

Received: 21-Jun-2013; Revised: 23-Jun2013; Accepted: 01-Aug-2013; Online first: 01-Oct-2013

Copyright: (c) 2013 Ezzat WM. This is an open-access article distributed under the terms of the Creative Commons Attribution License, which permits unrestricted use, distribution, and reproduction in any medium, provided the original author and source are credited.

Competing Interests: The authors have declared that no competing interests exist.
\end{abstract}

\begin{abstract}
We aimed to detect the validity of serum ATX as a diagnostic marker for liver fibrosis. Forty-eight males and 16 females were enrolled in the current study. Their ages ranged from 29-57 years with mean of 45.09 , all were chronically HCV infected. Laboratory assessment was done for all subjects in form of complete blood picture; liver function test; lipid profile and serum detection of ATX. Patients were grouped according to the stage of fibrosis into group 1: fibrosis score $0,1,2,3$; group 2: fibrosis score: 4, 5, 6. The mean values of ATX in all studied patients with chronic HCV infection was $63.02 \pm 36.29$ while that of healthy controls was $65.31 \pm 12.24$ without any significant difference. Surprisingly, mean values of ATX were higher among patients with group 1 but it did not reach the significant level. In each group of them, the differences between mean values of ATX among different grades of liver fibrosis were insignificant. It was also noticed that the mean values of ATX were higher among men than in women. It was concluded that Autotoxin might not be used as a useful diagnostic marker for liver fibrosis in Egyptian chronic HCV patients.
\end{abstract}

\section{Introduction}

The published Egyptian Demographic Health Survey (EDHS) in 2009 was a national probability sample of the resident Egyptian population. This report estimated an overall anti-HCV antibody prevalence of $14.7 \%$ [1].The major conclusion of Miller and Abu-Raddad, study is that the incidence of $\mathrm{HCV}$ in Egypt seems to be continuing at a rate of $\approx 6.9 / 1,000$ persons per year, indicative of possibly ongoing hyper epidemic transmission [2]

Chronic hepatitis $C$ is a slowly progressive inflammatory disease that can lead to cirrhosis with all its complications. Hepatic schistosomiasis in Egypt is commonly associated with HCV infection. Concurrent infection results in much more severe liver fibrosis than that seen in either disease alone. The prognosis of chronic liver disease depends on the progression of liver fibrosis. Liver biopsy is recommended as the gold standard method for determining fibrosis stage, prognosis and therapeutic indications in patients with chronic liver disease. However, liver biopsy is an invasive procedure associated with a risk of potentially serious complications [3]. Approximately $1-3 \%$ of patients require hospitalization for complications, and a quarter of them report pain after percutaneous liver biopsy. The diagnostic accuracy of liver biopsy for assessing liver fibrosis is influenced by the quality of the biopsy samples. In addition, there are several absolute or relative contraindications to liver biopsy, including severe coagulopathy. Among the possible alternatives, imaging is informative mainly for cirrhosis but not for lesser stages of fibrosis. In addition, it is nonquantitative and thus cannot track progression. A safe and non-invasive alternative to liver biopsy is therefore needed for assessing liver fibrosis in daily 
clinical practice [4]. Therefore, research has focused on the evaluation of noninvasive methods for the assessment of liver fibrosis. . Few serum markers such as hyaluronic acid, ferritin, vascular endothelial growth factor and soluble interleukin-2 receptor (sIL$2 \mathrm{R}$ ) have been reported to be useful in detecting fibrosis in liver disease $[5,6]$.

A previous study indicated that serum ATX level was significantly correlated with liver fibrosis stage and liver stiffness value [7]. Then, the performance of serum ATX level in the prediction of cirrhosis was better than serum hyaluronic acid and APRI. Autotaxin (ATX), a tumor cell motilitystimulating factor, regulates the blood concentrations of lysophosphatidic acid (LPA), an important and multi-functional bioactive lipid, through its lysophospholipase D activity (lysoPLD). The introduction of ATX measurements into clinical laboratory testing is urgently needed.

Autotaxin (ENPP-2) is a $125 \mathrm{kDa}$ secreted glycoprotein member of the nucleotide pyrophosphatase/phosphodiesterase (family of enzymes) that was first identified as a motility stimulating factor in melanoma cells [8]. It is an extracellular lysophospholipase $D$ that regulates the blood concentrations of lysophosphatidic acid (LPA), an important and multi-functional bioactive lipid from lysophosphatidylcholine (LPC). Both ATX and LPA have been shown to be involved in many cancers. However, the functional role of ATX and the regulation of ATX expression in human hepatocellular carcinoma (HCC) remain elusive [9]. ENPP-2 is normally widely expressed. It is upregulated in melanoma, glioblastoma, breast and lung carcinoma, follicular lymphoma and other cancers, promoting inflammation, proliferation, migration and angiogenesis, and is a potential target for treatment [10].

ENPP-2 stimulates tumor cell motility and enhances invasion and metastasis. ENPP-2 expression is elevated in reactive astrocytes adjacent to sites of neurotrauma, and in rheumatoid arthritis synoviocytes, Alzheimer's disease frontal cortex, and multiple sclerosis cerebrospinal fluids. It is thought to play a role in neuropathic pain [11]. Investigations to clarify the significance of ATX and LPA in liver fibrosis found that plasma LPA and serum ATX activity increased in patients with chronic hepatitis $C$ [12]. A strong correlation was observed between plasma LPA and serum ATX activity. Furthermore, plasma LPA and serum ATX activity were correlated with blood markers for liver fibrosis, such as serum hyaluronate and platelet counts. Plasma LPA and serum ATX activity increased in association with liver fibrosis stage histologically in these patients [13]. Because serum ATX activity and plasma LPA are well correlated with liver fibrosis stage histologically, both of these parameters merit consideration as novel markers of fibrosis [13].
The aim of this study is to detect validity of serum ATX as non-invasive diagnostic biomarker in the assessment of the liver fibrosis and moreover, to evaluate whether their serum levels are correlated with the histological severity of the related liver biopsies.

\section{Patients and Methods}

\section{Patients}

The study was conducted on 68 patients, 57 men and 11 women with documented chronic HCV infection, recruited from National Liver And Tropical Diseases Institute, Cairo. Chronic HCV diagnosis was based on elevated serum transaminase levels for at least six months and positive HCV antibody by the second-generation enzyme-linked immunosorbent assay and confirmed by detection of circulating HCV RNA using polymerase chain reaction (PCR). A liver biopsy specimen was taken from every patient. The study also included 20 healthy subjects, age and sex matched with the patients, as a control group. All controls were negative after anti HCV Ab screening test. Patients were initiated on treatment with subcutaneous pegylated interferon alfa-2a (180 $\mathrm{lg} /$ week) plus oral ribavirin (1000 or $1200 \mathrm{mg} /$ day).

Any patients with history of renal disorder, recent history of cardiovascular disease or patients with hepatitis $B$ infection or human immunodeficiency virus infection, autoimmune or metabolic liver diseases, diabetes mellitus, patients with body mass index $(\mathrm{BMI}) \geq 30 \mathrm{~kg} / \mathrm{m}^{2}$, patients not eligible for interferon therapy were excluded from the study. In addition, we excluded patients who were previously treated by any drug that may affect the results.

\section{Sample Collection}

After obtaining informed verbal consent, ten$\mathrm{ml}$ fasting blood samples collected in dry clean plastic tubes from both controls and patients. The blood were allowed to clot and sera were separated by centrifugation for $10 \mathrm{~min}$ at 3000 r.p.m using refrigerated centrifugation, divided into several aliquots and processed after conservation at $-20{ }^{\circ} \mathrm{C}$ until assayed. Samples confirmed to be collected without hemolysis. Major laboratory parameters, including blood sedimentation rate, liver and renal function tests, blood cell counts; random blood sugar, were evaluated at the same time points for all participants to exclude any organic disease or inflammation.

Sera from controls and patients subjected to the following investigations:

1. Complete blood picture.

2. Liver function tests including: Serum bilirubin level measured by using the commercially available kit from Bio-Merieux Company, France [14]. 
3. ALT, AST and Gamma glutamyl transferase measured by using the method recommended by the committee on enzymes of the Scandinavian Society for Clinical Chemistry and Clinical physiology, 1974 [15]. The test performed using commercially available kit from BoehringerMannhiem Company, Germany.

4. Cholesterol, triglycerides and Albumin.

5. Quantitative determination of Autotaxin was performed with commercially available Enzyme Immunoassay Kit [16].

\section{Principle of Autotaxin assay}

This assay employs the quantitative sandwich enzyme immunoassay technique. A monoclonal antibody specific for ENPP-2 has been pre-coated onto a microplate. Standards and samples are pipetted into the wells and any ENPP-2 present is bound by the immobilized antibody. After washing away any unbound substances, an enzyme-linked polyclonal antibody specific for ENPP-2 is added to the wells. Following a wash to remove any unbound antibody-enzyme reagent, a substrate solution is added to the wells and color develops in proportion to the amount of ENPP-2 bound in the initial step. The color development is stopped and the intensity of the color is measured [16].

\section{Histological evaluation}

Liver biopsy was obtained from all patients under ultrasound guidance. Biopsies were stained with hematoxylin and eosin and liver specific stains including van Giesonand Masson's Trichrome. All biopsies were evaluated by the same pathologist blinded to the clinical and laboratory criteria. Grading for the degree of necroinflammatory activity and staging for the extent of fibrosis were done according to the criteria of Ishak et al [17]. Patients were grouped according to the stage of fibrosis into group 1: fibrosis score $0,1,2,3$; group 2: fibrosis score: 4 , 5,6 . This study was carried out in accordance with the ethical guidelines. Informed consent from the patients was obtained for the use of the serum samples.

\section{Statistical analysis}

Data were presented as mean and standard deviation (SD) and percentage. The data were analyzed by SPSS version 14 (SPSS Inc., Chicago, IL, USA). The following tests of significance were used: t-test between means to analyze differences between means Differences between nominal variables were analyzed by $x^{2}$ tests. Two-tailed $p$ value $<0.05$ was considered statistically significant.

\section{Results}

Fifty-seven males and 11 females were enrolled in the current study. Their ages ranged from 29-57 years with mean of 45.09 years. Lab investigations in form of complete blood picture; liver function tests and lipid profile were recorded in Table 1 where all data were expressed as mean \pm SD.

As regards histopathological examination of liver biopsies, the mean values of Necroinflammation score was $9.17 \pm 4.61$. The frequencies of fibrosis stages $1-3$ and $4-5$ were $50 \%$, each. The frequencies of mild and moderate liver steatosis were $70.58 \%$ and $29.42 \%$ respectively as shown in Table 1.

Table 1: The demographic, clinical and biochemical data of the studied subjects. The data were expressed as mean \pm SD.

\begin{tabular}{|c|c|}
\hline Variables & Results \\
\hline Age & $45.09 \pm 6.07$ \\
\hline \multicolumn{2}{|l|}{ Sex } \\
\hline Males no (\%) & $57(83.8)$ \\
\hline Females no (\%) & $11(16.2)$ \\
\hline AST, IU/dl & $53.63 \pm 20.13$ \\
\hline ALT, IU/dl & $70.83 \pm 24.33$ \\
\hline $\mathrm{GGT}, \mathrm{IU} / \mathrm{dl}$ & $45.70 \pm 6.46$ \\
\hline PLT & $197340.0 \pm 26090.06$ \\
\hline $\mathrm{CHOL}, \mathrm{mg} / \mathrm{dl}$ & $299.57 \pm 72.87$ \\
\hline TRIG, mg/dl & $199.06 \pm 35.07$ \\
\hline LDL, mg/dl & $123.45 \pm 27.59$ \\
\hline $\mathrm{HDL}, \mathrm{mg} / \mathrm{dl}$ & $53.50 \pm 8.55$ \\
\hline $\mathrm{PC}, \%$ & $92.28 \pm 14.59$ \\
\hline INR & $0.95 \pm 0.63$ \\
\hline ALB, gm/dl & $4.62 \pm 4.41$ \\
\hline $\mathrm{PV}, \mathrm{mm}$ & $13.14 \pm 0.86$ \\
\hline \multicolumn{2}{|l|}{ Schistosomiasis: } \\
\hline Yes no (\%) & $35(51.5)$ \\
\hline No no (\%) & $33(48.5)$ \\
\hline Necroinflammation score & $9.17 \pm 4.61$ \\
\hline \multicolumn{2}{|l|}{ Grades of fibrosis: } \\
\hline $1-3$ no (\%) & $34(50)$ \\
\hline Liver steatosis & $34(50)$ \\
\hline Mild (\%) & $48(70.58)$ \\
\hline Moderate (\%) & $20(29.42)$ \\
\hline
\end{tabular}
Aspartate transaminase (AST), Alanine transaminase; (ALT), Gamma glutamyl
transaminase (GGT); Platelets (PLT); Hemoglobin (HGB); White blood cells (WBC); transaminase (GGT); Platelets (PLT); Hemoglobin (HGB); White blood cells (WBC);
Cholesterol (CHOL); Triglycerides (TRIG); Low density lipoprotein (LDL); High density Cholesterol (CHOL); Triglycerides (TRIG); Low density lipoprotein (LDL); High density
lipoprotein (HDL); Prothrombin concentration (PC); international normalization ratio (INR); Albumin (ALB); Portal vein (PV).

Comparison between group 1 patients with grades of liver fibrosis from $0-3$ and those group 2 with grades from 4-6 revealed that the group 2 had higher mean values of portal vein diameter and higher mean values of Necroinflammation score. Both differences were highly significant $(p<0.0001)$. Although group 2 had higher mean values of age and lipid profile than group 1, these differences did not reach the significant value.

Table 2: Comparison between two groups of patients as regards laboratory investigations.

\begin{tabular}{|c|c|c|c|c|}
\hline Variables & $\begin{array}{l}\mathrm{F} 0-\mathrm{F} 3 \\
\mathrm{~N}=34\end{array}$ & $\begin{array}{l}\mathrm{F} 4-\mathrm{F} 6 \\
\mathrm{~N}=34\end{array}$ & T test & $\begin{array}{c}P \\
\text { value }\end{array}$ \\
\hline Age & $44.56 \pm 6.03$ & $45.58 \pm 6.12$ & 0.834 & 0.406 \\
\hline AST, IU/dl & $53.29 \pm 20.97$ & $53.94 \pm 19.52$ & 0.161 & 0.873 \\
\hline ALT, IU/dl & $70.54 \pm 24.68$ & $71.10 \pm 24.24$ & 0.113 & 0.910 \\
\hline GGT, IU/dl & $46.10 \pm 5.95$ & $45.33 \pm 6.93$ & 0.599 & 0.550 \\
\hline PLT & $198210 \pm 27285$ & $196540 \pm 25176$ & 0.318 & 0.751 \\
\hline $\mathrm{CHOL}, \mathrm{mg} / \mathrm{dl}$ & $294.10 \pm 77.09$ & $304.62 \pm 69.11$ & 0.719 & 0.474 \\
\hline TRIG, mg/dl & $195.37 \pm 34.32$ & $202.46 \pm 35.75$ & 1.010 & 0.315 \\
\hline LDL, mg/dl & $121.75 \pm 24.29$ & $125.02 \pm 30.46$ & 0.590 & 0.556 \\
\hline $\mathrm{HDL}, \mathrm{mg} / \mathrm{dl}$ & $52.88 \pm 8.56$ & $54.08 \pm 8.57$ & 0.701 & 0.485 \\
\hline PC \% & $90.08 \pm 1.566$ & $94.31 \pm 13.35$ & 1.455 & 0.149 \\
\hline INR & $0.87 \pm 0.15$ & $1.03 \pm 0.86$ & 1.228 & 0.223 \\
\hline ALB, mgldl & $4.5 \pm 0.75$ & $4.21 \pm 0.55$ & .967 & 0.336 \\
\hline AST, IU/dl & $12.80 \pm 0.82$ & $13.45 \pm 0.80$ & 4.021 & $0.000^{*}$ \\
\hline $\begin{array}{l}\text { Necroinflammation } \\
\text { score }\end{array}$ & $5.29 \pm 3.69$ & $12.75 \pm 1.25$ & -13.750 & $0.000^{\star}$ \\
\hline ATX, $\mathrm{ng} / \mathrm{ml}$ & $71.22 \pm 39.33$ & $54.82 \pm 31.43$ & 1.899 & 0.062 \\
\hline
\end{tabular}

The mean values of ATX in all studied patients with chronic HCV infection was $63.02 \pm$ 36.29 , ranged from 21.80 to $134.52 \mathrm{ng} / \mathrm{ml}$ while that of 
healthy controls was $76.6 \pm 25.06$ ranged from 40.72 to $110.03 \mathrm{ng} / \mathrm{ml}$ without any significant difference.

Surprisingly, mean values of ATX were higher among patients with group 1 but it did not reach the significant level as shown in Table 2 and Fig. 1.

Table 3: Comparison between males and females as regards serum ATX values among different grades of fibrosis.

\begin{tabular}{lcc}
\hline Grades of fibrosis & Males & Females \\
& $\mathrm{N}=57$ & $\mathrm{~N}=11$ \\
\hline Grade 1 & $82.51 \pm 36.88$ & $62.30 \pm 26.99$ \\
Grade 2 & $70.40 \pm 37.62$ & $43.68 \pm 5.17$ \\
Grade 3 & $74.43 \pm 55.61$ & $33.14 \pm 0.00$ \\
Grade 4 & $48.67 \pm 29.05$ & $57.59 \pm 31.42$ \\
Grade 5 & $64.05 \pm 36.67$ & $43.37 \pm 23.10$ \\
Grade 6 & $41.67 \pm 0.00$ & 0.00 \\
F test & 1.236 & 0.441 \\
P value & 0.306 & 0.776 \\
\hline
\end{tabular}

Pattern of ATX expression in males and females were demonstrated in Table 3 , it was found that the highest mean values were found in grade 1 fibrosis among both males and females. In each group of them, the differences between mean values of ATX among different grades of liver fibrosis were insignificant. It is also noticed that the mean values of ATX were higher among men than in women in different grades of fibrosis except grade 4 as shown in Table 3, Fig. 2, and Fig. 3.

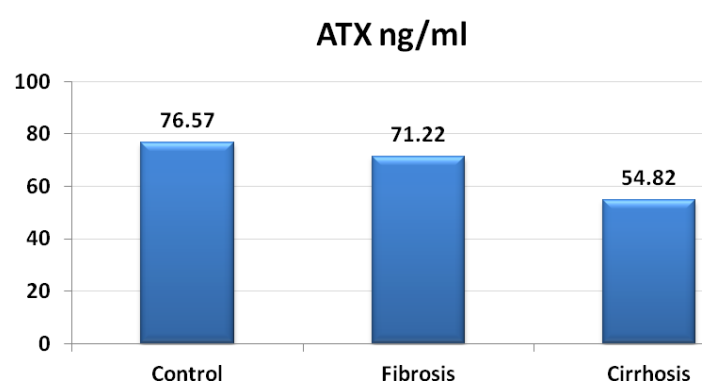

Figure 1: Mean values of ATX in studied groups of patients and controls.

The correlation between values of ATX and values of all studied parameters as that of blood picture; liver function tests; lipid profile; Necroinflammation score; grades of liver fibrosis revealed that ATX was not correlated to any of these parameters.

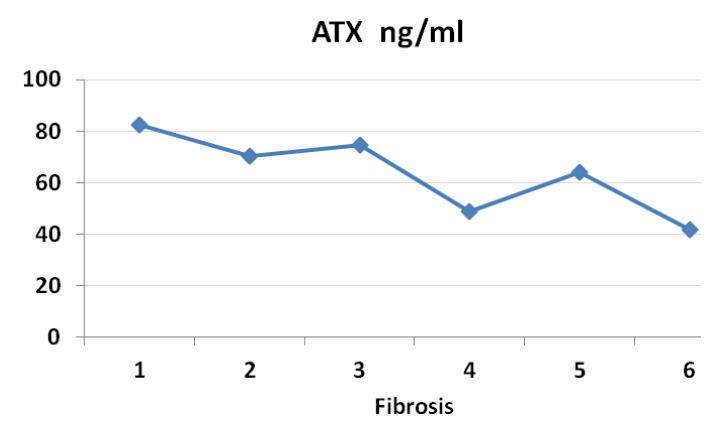

Figure 2: Mean values of ATX among males in different grades of fibrosis.

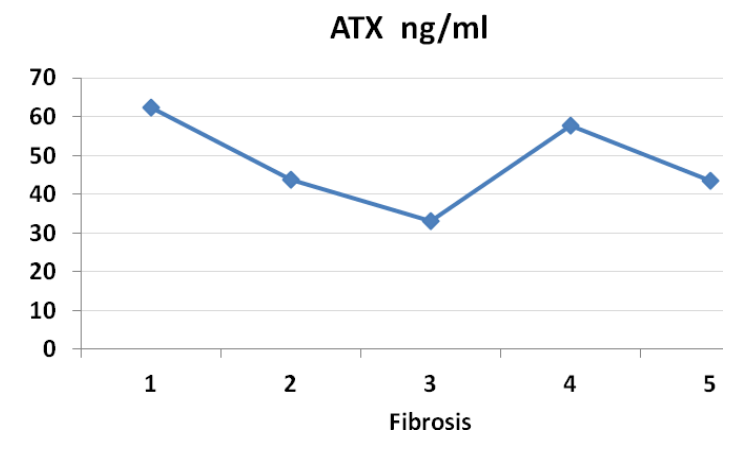

Figure 3: Mean values of ATX among females in different grades of fibrosis.

\section{Discussion}

It was found that ATX is not a diagnostic marker for liver fibrosis among studied patients with chronic HCV infection in the current study. These findings disagree with that of previous studies as Nakagawa and his colleagues, who concluded that serum ATX level was correlated significantly with liver stiffness, a novel reliable marker of liver fibrosis, being the second-best parameter in male (AUROC, 0.799) and in female (AUROC, 0.876) for prediction of significant fibrosis, and the best parameter in male (AUROC, 0.863) and the third-best parameter in female (AUROC, 0.872) for prediction of cirrhosis, both of which were judged by liver stiffness [18]. In an experimental study, Watanabe and his colleagues suggested that Plasma LPA level and serum ATX activity are increased in various liver injuries in relation to their severity. At the same time, they recommended that whether increased ATX and LPA in the blood in liver injury is simply a result or also a cause of the injury should be further clarified [13].

Several questions concerning circulating ATX remain to be answered, including its tissue origin and metabolic fate, although recent evidence indicates that ATX is rapidly cleared from the circulation by liver sinusoidal endothelial cells [19]. Circulating ATX and LPA, of course, do not reflect the levels in intercellular spaces, since many different cell types produce ATX locally while the LPC substrate level in interstitial fluids is much lower than that in plasma. Another key question concerns how ATX activity is regulated under (patho) physiological conditions. Interestingly, ATX binds to activated lymphocytes and platelets in an integrin-dependent manner, which could lead to altered catalytic activity and serve as a mechanism for localized LPA production at sites of inflammation and injury. Our different findings may be refereed to genetic variation between the studied Egyptian population and other foreign patients in previous studies. The human ATX gene is organized in 27 exons, and three alternative splicing isoforms of ATX, 
named $a, b$, and $c$, have been reported [20]. Compared to the isoform $b$, the isoform a contains $a$ 52 residue-insertion (exon 12) in the catalytic domain, and the isoform c contains a 25-residue insertion (exon 21) close to the nuclease-like domain. $\mathrm{Li}$ and his colleagues confirmed that the activation of PKR, c-Jun N-terminal kinase JNK, and p38 mitogen activated protein kinase MAPK was required for the ATX induction. The LPS-induced ATX in THP-1 cells was characterized as the $b$ isoform [21]. So that molecular basis for ATX serum expression may be changed between different populations. Hepatic schistosomiasis among Egyptian patients with chronic $\mathrm{HCV}$ may be another contributing factor in contradiction of our findings. in the same context, it was proved that MAPKs are pivotal transmitters of extracellular signals such as hormones, cytokines, growth factors, and various environmental stress signals [22]. The MAPK family has three major subfamilies: ERK, P38, and JNK, and the P38 and JNK pathways are involved in hepatic satellite cells (HSC) transformation [23] and in the regulation of the complex life cycle and host-parasite interactions of schistosomiasis. While schistosomiasis is associated with an imbalance in T helper 1 (Th1)/Th2 cytokines [24], differentiation of CD4+ T cells into Th1 or Th2 lymphocyte population depends to a great extent upon the relative abundance of various cytokines during the priming of the antigen-specific lymphocyte population by antigen-presenting cells [25, 26]. Collectively, different genetic predisposition; different environmental stress and co morbid added schistosomiasis, all may share in different pattern of immune expression that may reflect on serum ATX expression.

What mechanism responsible for increased plasma LPA and serum ATX activity in liver fibrosis? Factors involved with increasing serum ATX activity are likely primary because plasma LPA is essentially regulated by ATX [13] To explain increased serum ATX activity, two possibilities should be considered (although the origin and fate of serum ATX have not been fully elucidated): increased ATX production as a result of liver fibrosis or reduction in ATX clearance. In carbon tetrachloride-induced fibrotic rat livers, ATX mRNA expression was unaltered [13], i.e., production was not increased. Increased serum ATX activity was observed in $70 \%$ hepatectomized rats, as early as three hours post-surgery, and maintained for $24 \mathrm{~h}$ [13]. These findings suggest that increased serum ATX activity may be caused by a reduction in clearance. As such, the liver may not be the major site of ATX production, but the site of ATX clearance. In agreement, ATX was subsequently shown to be cleared from the circulation within minutes, taken up, and degraded in liver sinusoidal endothelial cells [18]. During the process of liver fibrosis, liver sinusoidal endothelial cells are known to undergo phenotypic changes with a loss of various receptors and sinusoidal endothelial fenestrae causing the capillarization of the sinusoids, thereby impairing the uptake of various substances.

Although previous in vitro findings have suggested a link between LPA and liver fibrosis in vivo, whether LPA plays a primary role in the Pathogenesis of liver fibrosis has remained uncertain. No phenotypic changes in livers of LPA receptordeficient mice have been demonstrated [27]. No modulations in liver fibrosis have so far been reported in these mice after liver injury. Of note, previous evidence has demonstrated relatively low gene expression of LPA receptors in Liver [28], suggesting that the roles of LPA may be limited in liver fibrosis.

In the current study we measured serum ATX not LPA because it was suggested that LPA can increase markedly after sample preparation unless temperature is strictly controlled [29]. This finding may result from the presence of synthetic ATX and its substrate lysophosphatidyl choline that can lead to abundant production of LPA in plasma [29]. Second, LPA is released from platelets, among other sources, and LPA should be measured in plasma to evaluate its clinical significance [30]. On the other hand, ATX activity can be measured in serum and is temperature stable [31].

Nakagawa and his colleagues talked about gender bias of ATX expression but they did not give us explanation about this bias. In the current study, we noticed that in both males and females the differences between mean values of ATX among different grades of liver fibrosis were insignificant. It is also noticed that the mean values of ATX were higher among men than in women in different grades of fibrosis except grade 4 fibrosis. Gender bias of ATX may be referred to hormonal effects as it may be used as a marker for ovarian cancer [32].

ATX was originally discovered in conditioned medium from A2058 human melanoma cell cultures and was characterized as a stimulator of cell migration [8]. Since then, ATX has been speculated to play a role in cancer invasion or metastasis as an autocrine motility factor [34]. However, the exact pathophysiological significanceof ATX remained unknown until the discovery of its lysoPLD activity [33, 34] so, it was evident that ATX is not a specific marker for liver injury and this may be reflected on its serum expression in relation to liver fibrosis. Moreover, we cannot depend on it in diagnosis or prediction of liver fibrosis.

In conclusion, autotaxin may be not a diagnostic marker for liver fibrosis in Egyptian chronic HCV patients. Liver biopsy is recommended as the gold standard method for determining fibrosis.

\section{References}

1. Zanaty F, Way A. Egypt Demographic and Health Survey 2008. Egyptian: Ministry of Health (El-Zanaty and Associates and Macro International, Cairo). 2009: :pp 431. 
2. Miller FD, Abu-Raddad LJ. Evidence of intense ongoing endemic transmission of hepatitis $C$ virus in Egypt. Proceedings of the National Academy of Sciences. 2010;107(33):14757-62.

3. Palmeri ML, Wang MH, Rouze NC, Abdelmalek MF, Guy CD, Moser B, et al. Noninvasive evaluation of hepatic fibrosis using acoustic radiation force-based shear stiffness in patients with nonalcoholic fatty liver disease. Journal of hepatology. 2011;55(3):666-72.

4. Boursier J, Isselin G, Fouchard-Hubert I, Oberti F, Dib N, Lebigot $\mathrm{J}$, et al. Acoustic radiation force impulse: a new ultrasonographic technology for the widespread noninvasive diagnosis of liver fibrosis. European journal of gastroenterology \& hepatology. 2010;22(9):1074-84.

5. Ali Gaballa A.A.; Ayman M. Fouad; Mona I. Nabih; Halla M Ragab and Nabila A. Abdalla. Vascular Endothelial growth facto expression in plasma of patients with liver cirrhosis and hepatocellular carcinoma. Kasr El Aini Medical Journal. 2003;9(6):361-370

6. Mohab H. Halim., Nabila A. Abdalla, Halla M. Ragab, Nouran E Ghandour. Role of non invasive biomarkers in the assessment of liver condition in chronic hepatitis $\mathrm{C}$ Egyptian patients and if they correlate with the severity of liver affection. Med J Cairo Univ. 2005 73( 4):49-62.

7. Moolenaar WH, van Meeteren LA, Giepmans BN. The ins and outs of lysophosphatidic acid signaling. BioEssays : news and reviews in molecular, cellular and developmental biology. 2004; 26 (8):870-81.

8. Stracke ML, Krutzsch HC, Unsworth EJ, Arestad A, Cioce V Schiffmann $E$, et al. Identification, purification, and partial sequence analysis of autotaxin, a novel motility-stimulating protein. The Journal of biological chemistry. 1992;267(4):2524-9.

9. Wu JM, Xu Y, Skill NJ, Sheng H, Zhao Z, Yu M, et al. Autotaxin expression and its connection with the TNF-alpha-NF-kappaB axis in human hepatocellular carcinoma. Molecular cancer. 2010;9:71.

10. Nakanaga K, Hama K, Aoki J. Autotaxin--an LPA producing enzyme with diverse functions. Journal of biochemistry. 2010;148(1):13-24

11. Van Meeteren LA, Moolenaar WH. Regulation and biologica activities of the autotaxin-LPA axis. Progress in lipid research 2007;46(2):145-60.

12. Mills GB, Moolenaar WH. The emerging role of lysophosphatidic acid in cancer. Nature reviews Cancer. 2003;3(8):582-91.

13. Watanabe $N$, lkeda $H$, Nakamura $K$, Ohkawa $R$, Kume $Y$ Tomiya $\mathrm{T}$, et al. Plasma lysophosphatidic acid level and serum autotaxin activity are increased in liver injury in rats in relation to its severity. Life sciences. 2007;81(12):1009-15.

14. Winsten S, Cehelyk B. A rapid micro diazo technique for measuring total bilirubin. Clin Chim Acta. 1969; 25: 441-6.

15. Committee on enzymes of the Scandinavian Society for Clinica Chemistry and Clinical physiology. Recommended methods for the determination of four enzymes in blood. Scand J Lab Invest. 1974; 197(3): 291-299.

16. Butler JE, Spradling JE, Suter M, Dierks SE, Heyermann H, Peterman JH. The immunochemistry of sandwich ELISAs--I. The binding characteristics of immunoglobulins to monoclonal and polyclonal capture antibodies adsorbed on plastic and their detection by symmetrical and asymmetrical antibody-enzyme conjugates. Molecular immunology. 1986;23(9):971-82.

17. Ishak K, Baptista A, Bianchi L, Callea F, De Groote J, Gudat F, et al. Histological grading and staging of chronic hepatitis. Journal of hepatology. 1995;22(6):696-9.

18. Nakagawa $H$, Ikeda $H$, Nakamura $K$, Ohkawa $R$, Masuzaki $R$, Tateishi R, et al. Autotaxin as a novel serum marker of liver fibrosis. Clinica chimica acta; international journal of clinical chemistry. 2011;412(13-14):1201-6.
19. Jansen S, Andries M, Vekemans K, Vanbilloen H, Verbruggen A, Bollen M. Rapid clearance of the circulating metastatic factor autotaxin by the scavenger receptors of liver sinusoidal endothelial cells. Cancer letters. 2009;284(2):216-21.

20. Giganti A, Rodriguez M, Fould B, Moulharat N, Coge F Chomarat $\mathrm{P}$, et al. Murine and human autotaxin alpha, beta, and gamma isoforms: gene organization, tissue distribution, and biochemical characterization. The Journal of biological chemistry. 2008;283(12):7776-89.

21. Li S, Zhang J. Lipopolysaccharide induces autotaxin expression in human monocytic THP-1 cells. Biochemical and biophysical research communications. 2009;378(2):264-8

22. Widmann C, Gibson S, Jarpe MB, Johnson GL. Mitogenactivated protein kinase: conservation of a three-kinase module from yeast to human. Physiological reviews. 1999;79(1):143-80.

23. Reeves HL, Dack CL, Peak M, Burt AD, Day CP. Stressactivated protein kinases in the activation of rat hepatic stellate cells in culture. Journal of hepatology. 2000;32(3):465-72.

24. Yu L, Sun $X$, Yang F, Yang J, Shen J, Wu Z. Inflammatory cytokines IFN-gamma, IL-4, IL-13 and TNF-alpha alterations in schistosomiasis: a meta-analysis. Parasitology research. 2012;110(4):1547-52.

25. O'Garra A. Cytokines induce the development of functionally heterogeneous T helper cell subsets. Immunity. 1998;8(3):275-83.

26. Seder RA, Paul WE. Acquisition of lymphokine-producing phenotype by CD4+ $\mathrm{T}$ cells. Annual review of immunology. 1994;12:635-73.

27. Choi JW, Lee CW, Chun J. Biological roles of lysophospholipid receptors revealed by genetic null mice: an update. Biochimica et biophysica acta. 2008;1781(9):531-9

28. Choi JW, Herr DR, Noguchi K, Yung YC, Lee CW, Mutoh T, et al. LPA receptors: subtypes and biological actions. Annual review of pharmacology and toxicology. 2010;50:157-86.

29. Nakamura K, Ohkawa R, Okubo S, Tozuka M, Okada M, Aoki $S$, et al. Measurement of lysophospholipase D/autotaxin activity in human serum samples. Clinical biochemistry. 2007;40(3-4):274-7.

30. Baker DL, Morrison P, Miller B, Riely CA, Tolley B, Westermann $A M$, et al. Plasma lysophosphatidic acid concentration and ovarian cancer. JAMA : the journal of the American Medical Association. 2002;287(23):3081-2

31. Li H, Wang D, Zhang H, Kirmani K, Zhao Z, Steinmetz R, et al. Lysophosphatidic acid stimulates cell migration, invasion, and colony formation as well as tumorigenesis/metastasis of mouse ovarian cancer in immunocompetent mice. Molecular cancer therapeutics. 2009;8(6):1692-701.

32. Stracke ML, Clair T, Liotta LA. Autotaxin, tumor motilitystimulating exophosphodiesterase. Advances in enzyme regulation. 1997;37:135-44

33. Tokumura A, Majima E, Kariya Y, Tominaga K, Kogure K, Yasuda K, et al. Identification of human plasma lysophospholipase $\mathrm{D}$, a lysophosphatidic acid-producing enzyme, as autotaxin, a multifunctional phosphodiesterase. The Journal of biological chemistry. 2002;277(42):39436-42

34. Umezu-Goto M, Kishi Y, Taira A, Hama K, Dohmae N, Takio K, et al. Autotaxin has lysophospholipase D activity leading to tumor cell growth and motility by lysophosphatidic acid production. The Journal of cell biology. 2002;158(2):227- 233. 